\title{
Construction of the type 2 poly-Frobenius-Genocchi polynomials with their certain applications
}

\author{
Ugur Duran' ${ }^{1}$, Mehmet Acikgoz ${ }^{2}$ and Serkan Araci $^{3^{*}}$ (D)
}

\section{"Correspondence:}

serkan.araci@hku.edu.tr

${ }^{3}$ Department of Economics, Faculty

of Economics, Administrative and

Social Sciences, Hasan Kalyoncu

University, TR-27410 Gaziantep,

Turkey

Full list of author information is

available at the end of the article

\begin{abstract}
Kim and Kim (Russ. J. Math. Phys. 26(1):40-49, 2019) have studied the type 2 poly-Bernoulli polynomials. Inspired by their work, we consider a new class of the Frobenius-Genocchi polynomials, which is called the type 2 poly-Frobenius-Genocchi polynomials, by means of the polyexponential function. We also derive some new relations and properties including the Stirling numbers of the first and second kinds. In a special case, we give a relation between the type 2 poly-Frobenius-Genocchi polynomials and Bernoulli polynomials of order $k$. Moreover, motivated by the definition of the unipoly-Bernoulli polynomials given in (Kim and Kim in Russ. J. Math. Phys. 26(1):40-49, 2019), we introduce the unipoly-Frobenius-Genocchi polynomials via a unipoly function and give multifarious properties including derivative and integral properties. Furthermore, we provide a correlation between the unipoly-Frobenius-Genocchi polynomials and the classical Frobenius-Genocchi polynomials.
\end{abstract}

MSC: 11B83; 11S80;05A19

Keywords: Polylogarithm function; Polyexponential function; Frobenius-Genocchi polynomials; Poly-Frobenius-Genocchi polynomials

\section{Introduction and preliminaries}

Special polynomials have their origin in the solution of the differential equations (or partial differential equations) under some conditions. Special polynomials can be defined in various ways such as by generating functions, by recurrence relations, by $p$-adic integrals in the sense of fermionic and bosonic, by degenerate versions, etc.

Kim and Kim have introduced polyexponential function in [12] and its degenerate version in $[14,16]$. By making use of these functions, they have introduced a new class of some special polynomials. This idea provides a powerful tool in order to define new types of special numbers and polynomials by making use of polyexponential function and degenerate polyexponential function. It is worthy to note that the notion of polyexponential function forms a special class of polynomials because of their great applicability. The importance of these polynomials would be to find applications in analytic number theory, applications in classical analysis and statistics.

(c) The Author(s) 2020. This article is licensed under a Creative Commons Attribution 4.0 International License, which permits use sharing, adaptation, distribution and reproduction in any medium or format, as long as you give appropriate credit to the original author(s) and the source, provide a link to the Creative Commons licence, and indicate if changes were made. The images or other third party material in this article are included in the article's Creative Commons licence, unless indicated otherwise in a credit line to the material. If material is not included in the article's Creative Commons licence and your intended use is not permitted by statutory regulation or exceeds the permitted use, you will need to obtain permission directly from the copyright holder. To view a copy of this licence, visit http://creativecommons.org/licenses/by/4.0/. 
Throughout the paper we make use of the following notations: $\mathbb{N}:=\{1,2,3, \ldots\}$ and $\mathbb{N}_{0}=$ $\mathbb{N} \cup\{0\}$. Here, as usual, $\mathbb{Z}$ denotes the set of integers, $\mathbb{R}$ denotes the set of real numbers, and $\mathbb{C}$ denotes the set of complex numbers.

The Bernoulli $B_{n}(x)$, Euler $E_{n}(x)$, and Genocchi $G_{n}(x)$ polynomials are defined by the following exponential generating functions, respectively:

$$
\frac{t}{e^{t}-1} e^{x t}=\sum_{n=0}^{\infty} B_{n}(x) \frac{t^{n}}{n !} \quad(|t|<2 \pi), \quad \frac{2}{e^{t}+1} e^{x t}=\sum_{n=0}^{\infty} E_{n}(x) \frac{t^{n}}{n !} \quad(|t|<\pi)
$$

and

$$
\frac{2 t}{e^{t}+1} e^{x t}=\sum_{n=0}^{\infty} G_{n}(x) \frac{t^{n}}{n !} \quad(|t|<\pi) .
$$

One may see the references $[2-7,9-11,13,17,23]$ for the various applications of Bernoulli, Euler, and Genocchi polynomials.

Frobenius studied the polynomials $F_{n}(x \mid u)$ given by

$$
\frac{1-u}{e^{t}-u} e^{x t}=\sum_{n=0}^{\infty} F_{n}(x \mid u) \frac{t^{n}}{n !} \quad\left(u \in \mathbb{C} \backslash\{1\} ; e^{t} \neq u\right)
$$

When $u=-1$, it becomes

$$
F_{n}(x \mid-1)=E_{n}(x) .
$$

Owing to relationship with the Euler polynomials as well as their important properties, and in the honor of Frobenius, the aforementioned polynomials denoted by $F_{n}(x \mid u)$ are called the Frobenius-Euler polynomials, $c f$. [18].

Parallel to (2), Yaşar and Özarslan [25] introduced the Frobenius-Genocchi polynomials $G_{n}^{F}(x ; u)$ given by

$$
\frac{(1-u) t}{e^{t}-u} e^{x t}=\sum_{n=0}^{\infty} G_{n}^{F}(x ; u) \frac{t^{n}}{n !}
$$

since

$$
G_{n}^{F}(x ;-1)=G_{n}(x)
$$

In the case $x=0$ in (3), $G_{n}^{F}(0 ; u):=G_{n}^{F}(u)$ stands for the Frobenius-Genocchi numbers. Several recurrence relations and differential equations are also investigated in [25].

Khan and Srivastava [11] introduced a new class of the generalized Apostol type Frobenius-Genocchi polynomials and investigated some properties and relations including implicit summation formulae and various symmetric identities. Moreover, a relation in between Array-type polynomials, Apostol-Bernoulli polynomials, and generalized Apostol-type Frobenius-Genocchi polynomials is also given in [11]. Wani et al. [24] considered Gould-Hopper based Frobenius-Genocchi polynomials and summation formulae and an operational rule for these polynomials. 
The Bernoulli polynomials of the second kind are defined by means of the following generating function:

$$
\sum_{n=0}^{\infty} b_{n}(x) \frac{t^{n}}{n !}=\frac{t}{\log (1+t)}(1+t)^{x}
$$

When $x=0, b_{n}(0):=b_{n}$ are called Bernoulli numbers of the second kind, $c f$. [16].

It is well known from (4) that

$$
\frac{t}{\log (1+t)}(1+t)^{x-1}=\sum_{n=0}^{\infty} B_{n}^{(n)}(x) \frac{t^{n}}{n !}
$$

where $B_{n}^{(k)}(x)$ are the Bernoulli polynomials of order $k$ which are given by the following generating function:

$$
\sum_{n=0}^{\infty} B_{n}^{(k)}(x) \frac{t^{n}}{n !}=\left(\frac{t}{e^{t}-1}\right)^{k} e^{x t}
$$

By (4) and (5), it is clear that $B_{n}^{(n)}(x+1)=b_{n}(x)$, see [16].

Very recently, Kim and Kim [12] performed to generalize the Bernoulli polynomials by using the polyexponential function

$$
e_{k}(t)=\sum_{n=1}^{\infty} \frac{t^{n}}{(n-1) ! n^{k}}
$$

as inverse to the polylogarithm function

$$
\operatorname{Li}_{k}(t)=\sum_{n=1}^{\infty} \frac{t^{n}}{n^{k}} \quad(|t|<1 ; k \in \mathbb{Z})
$$

given by

$$
\sum_{n=0}^{\infty} \beta_{n}^{(k)}(x) \frac{t^{n}}{n !}=\frac{e_{k}(\log (1+t))}{e^{t}-1} e^{x t} \quad(k \in \mathbb{Z})
$$

Upon setting $x=0$ in (8), $\beta_{n}^{(k)}(0):=\beta_{n}^{(k)}$ are called the type 2 poly-Bernoulli numbers.

Since

$$
e_{1}(t)=e^{t}-1
$$

we have

$$
\beta_{n}^{(1)}(x):=B_{n}(x)
$$

Kim and Kim [12] also introduced a unipoly function $u_{k}(x \mid p)$ attached to $p$ being any arithmetic function that is a real- or complex-valued function defined on the set of positive 
integers as follows:

$$
u_{k}(x \mid p)=\sum_{n=1}^{\infty} \frac{p(n)}{n^{k}} x^{n} \quad(k \in \mathbb{Z})
$$

It follows from (9) that

$$
u_{k}(x \mid 1)=\sum_{n=1}^{\infty} \frac{x^{n}}{n^{k}}=L i_{k}(x)
$$

is the polylogarithm function as given in (7). The unipoly function attached to $p$ satisfies the following properties for $k \geq 2$ :

$$
\frac{d}{d x} u_{k}(x \mid p)=\frac{1}{x} u_{k-1}(x \mid p)
$$

and

$$
u_{k}(x \mid p)=\int_{0}^{x} \frac{1}{t} \underbrace{\int_{0}^{t} \frac{1}{t} \cdots \int_{0}^{t} \frac{1}{t}}_{(k-2) \text { times }} u_{1}(x \mid p) d t d t \cdots d t .
$$

By means of the unipoly function, Kim and Kim [12] defined unipoly-Bernoulli polynomials as follows:

$$
\sum_{n=0}^{\infty} B_{n, p}^{(k)}(x) \frac{t^{n}}{n !}=\frac{u_{k}\left(1-e^{-t} \mid p\right)}{1-e^{-t}} e^{x t}
$$

They provided several formulae and relations for these polynomials, see [12].

Kwon and Jang [20] defined the type 2 poly-Apostol-Bernoulli polynomials and provided some properties for them. Moreover, by making use of a unipoly function, they considered the type 2 unipoly-Apostol-Bernoulli numbers and proved some basic properties.

The Stirling numbers of the first kind $S_{1}(n, k)$ and the Stirling numbers of the second kind $S_{2}(n, k)$ are defined by means of the following generating functions:

$$
\frac{(\log (1+t))^{k}}{k !}=\sum_{n=0}^{\infty} S_{1}(n, k) \frac{t^{n}}{n !}
$$

and

$$
\frac{\left(e^{t}-1\right)^{k}}{k !}=\sum_{n=0}^{\infty} S_{2}(n, k) \frac{t^{n}}{n !} .
$$

From (11), we get the following relations for $n \in \mathbb{N}_{0}$ :

$$
(x)_{n}=\sum_{k=0}^{n} S_{1}(n, k) x^{k}
$$


and

$$
x^{n}=\sum_{k=0}^{n} S_{1}(n, k)(x)_{k},
$$

where $(x)_{0}=1$ and $(x)_{n}=x(x-1)(x-2) \cdots(x-n+1), c f$. $[2,3,18]$.

An outline of this paper is as follows. Section 2 deals with the construction of a class of new generating functions for the Frobenius-Genocchi polynomials, called the type 2 poly-Frobenius-Genocchi polynomials, by means of the polyexponential function and also provides some useful relations and properties. In addition, this section shows that the type 2 poly-Frobenius-Genocchi polynomials equal a linear combination of the classical Frobenius-Genocchi polynomials and Stirling numbers of the first kind. Section 3 gives the definition of the unipoly-Frobenius-Genocchi polynomials by means of a unipoly function and includes several properties including derivative and integral properties. Furthermore, a correlation between the unipoly-Frobenius-Genocchi polynomials and the classical Frobenius-Genocchi polynomials is stated in Sect. 3. In the last section, the results obtained in this paper are examined.

\section{The type 2 poly-Frobenius-Genocchi polynomials}

Motivated and inspired by the definition of the type 2 poly-Bernoulli polynomials in (8) introduced by Kim and Kim [12], in this paper, we consider the following Definition 2.1 by means of the polyexponential function.

Definition 2.1 Let $k \in \mathbb{Z}$. The type 2 poly-Frobenius-Genocchi polynomials are defined via the following exponential generating function (in a suitable neighborhood of $t=0$ ) including the polyexponential function:

$$
\sum_{n=0}^{\infty} G_{n}^{(F, k)}(x ; u) \frac{t^{n}}{n !}=\frac{e_{k}(\log (1+(1-u) t))}{e^{t}-u} e^{x t} .
$$

At the value $x=0$ in $(13), G_{n}^{(F, k)}(0 ; u):=G_{n}^{(F, k)}(u)$ will be called type 2 poly-FrobeniusGenocchi numbers.

Remark 2.1 Taking $k=1$ in (13) yields $G_{n}^{(F, 1)}(x ; u):=G_{n}^{F}(x ; u)$.

Remark 2.2 Taking $k=1$ and $u=-1$ in (13) gives $G_{n}^{(F, 1)}(x ;-1):=G_{n}(x)$, $[15,19]$.

By Definition 2.1, we consider that

$$
\begin{aligned}
\sum_{n=0}^{\infty} G_{n}^{(F, k)}(x ; u) \frac{t^{n}}{n !} & =\frac{e_{k}(\log (1+(1-u) t))}{e^{t}-u} e^{x t} \\
& =\left(\sum_{n=0}^{\infty} G_{n}^{(F, k)}(u) \frac{t^{n}}{n !}\right)\left(\sum_{n=0}^{\infty} x^{n} \frac{t^{n}}{n !}\right) \\
& =\sum_{n=0}^{\infty}\left(\sum_{l=0}^{n}\left(\begin{array}{l}
n \\
l
\end{array}\right) G_{n-l}^{(F, k)}(u) x^{l}\right) \frac{t^{n}}{n !} .
\end{aligned}
$$

Hence, we give the following theorem. 
Theorem 2.1 The following relation

$$
G_{n}^{(F, k)}(x ; u)=\sum_{l=0}^{n}\left(\begin{array}{l}
n \\
l
\end{array}\right) G_{n-l}^{(F, k)}(u) x^{l}
$$

is valid for $k \in \mathbb{Z}$ and $n \in \mathbb{N}_{0}$.

A relation between the type 2 poly-Frobenius-Genocchi polynomials and the classical Frobenius-Genocchi polynomials is stated in the following theorem.

Theorem 2.2 For $k \in \mathbb{Z}$ and $n \in \mathbb{N}_{0}$, we have

$$
G_{n}^{(F, k)}(x ; u)=\sum_{l=0}^{n} \sum_{m=0}^{l}\left(\begin{array}{l}
n \\
l
\end{array}\right) \frac{1}{(m+1)^{k-1}} S_{1}(l+1, m+1) \frac{(1-u)^{l}}{l+1} G_{n-l}^{F}(x ; u) .
$$

Proof From (6), (11), and (13), we observe that

$$
\begin{aligned}
& \sum_{n=0}^{\infty} G_{n}^{(F, k)}(x ; u) \frac{t^{n}}{n !} \\
& \quad=\frac{e_{k}(\log (1+(1-u) t))}{e^{t}-u} e^{x t} \\
& \quad=\frac{e^{x t}}{e^{t}-u} \sum_{m=1}^{\infty} \frac{(\log (1+(1-u) t))^{m}}{(m-1) ! m^{k}} \\
& =\frac{e^{x t}}{e^{t}-u} \sum_{m=0}^{\infty} \frac{1}{(m+1)^{k}} \frac{(\log (1+(1-u) t))^{m+1}}{m !} \\
& =\frac{e^{x t}}{e^{t}-u} \sum_{m=0}^{\infty} \frac{1}{(m+1)^{k-1}} \sum_{n=m+1}^{\infty} S_{1}(n, m+1) \frac{((1-u) t)^{n}}{n !} \\
& =\frac{(1-u) t}{e^{t}-u} e^{x t} \sum_{m=0}^{\infty} \frac{1}{(m+1)^{k-1}} \sum_{n=m}^{\infty} S_{1}(n+1, m+1) \frac{(1-u)^{n}}{n+1} \frac{t^{n}}{n !} \\
& =\sum_{n=0}^{\infty} G_{n}^{F}(x ; u) \frac{t^{n}}{n !} \sum_{n=0}^{\infty}\left(\sum_{m=0}^{n} \frac{1}{(m+1)^{k-1}} \sum_{n=m}^{\infty} S_{1}(n+1, m+1) \frac{(1-u)^{n}}{n+1}\right) \frac{t^{n}}{n !} \\
& \quad=\sum_{n=0}^{\infty}\left(\sum_{l=0}^{n} \sum_{m=0}^{l}\left(\begin{array}{l}
n \\
l
\end{array}\right) \frac{1}{(m+1)^{k-1}} S_{1}(l+1, m+1) \frac{(1-u)^{l}}{l+1} G_{n-l}^{F}(x ; u)\right) \frac{t^{n}}{n !}
\end{aligned}
$$

which means the asserted result in (15).

The immediate results of Theorem 2.2 are stated in what follows.

Corollary 2.1 For $k \in \mathbb{Z}$ and $n \in \mathbb{N}_{0}$, we have

$$
G_{n}^{(F, k)}(u)=\sum_{l=0}^{n} \sum_{m=0}^{l}\left(\begin{array}{l}
n \\
l
\end{array}\right) \frac{1}{(m+1)^{k-1}} S_{1}(l+1, m+1) \frac{(1-u)^{l}}{l+1} G_{n-l}^{F}(u) .
$$


Corollary 2.2 Taking $k=1$ in Theorem 2.2 gives

$$
G_{n}^{F}(x ; u)=\sum_{l=0}^{n} \sum_{m=0}^{l}\left(\begin{array}{l}
n \\
l
\end{array}\right) S_{1}(l+1, m+1) \frac{(1-u)^{l}}{l+1} G_{n-l}^{F}(x ; u) .
$$

Corollary 2.3 Taking $k=1$ and $u=-1$ in Theorem 2.2 reduces

$$
G_{n}(x)=\sum_{l=0}^{n} \sum_{m=0}^{l} \frac{\left(\begin{array}{l}
n \\
l
\end{array}\right)}{l+1} 2^{l} S_{1}(l+1, m+1) G_{n-l}(x)
$$

and

$$
\sum_{l=1}^{n} \sum_{m=0}^{l} \frac{\left(\begin{array}{l}
n \\
l
\end{array}\right)}{l+1} 2^{l} S_{1}(l+1, m+1) G_{n-l}(x)=0 .
$$

Let $s \in \mathbb{C}$ and $k \in \mathbb{Z}$ with $k \geq 1$. We consider the function $\eta_{k, u}$ by the representation of an improper integral as follows:

$$
\eta_{k, u}(s):=\frac{(1-u)^{s-1}}{\Gamma(s)} \int_{0}^{\infty} \frac{t^{s-1}}{e^{t}-u} e_{k}(\log (1+(1-u) t)) d t,
$$

where $\Gamma(s)$ is the well-known gamma function defined by

$$
\Gamma(s)=\int_{0}^{\infty} t^{s-1} e^{t} d t \quad(\Re(s)>0) .
$$

By (17), we observe that

$$
\begin{aligned}
\eta_{1, u}(s) & =\frac{(1-u)^{s-1}}{\Gamma(s)} \int_{0}^{\infty} \frac{t^{s-1}}{e^{t}-u} e_{1}(\log (1+(1-u) t)) d t \\
& =\frac{(1-u)^{s}}{\Gamma(s)} \int_{0}^{\infty} \frac{t^{s}}{e^{t}-u} d t \\
& =\frac{(1-u)^{s}}{\Gamma(s)} \int_{0}^{\infty} \frac{t^{s} e^{-t}}{1-u e^{-t}} d t \\
& =(1-u)^{s} \Phi(u, s+1,1),
\end{aligned}
$$

where

$$
\begin{aligned}
\Phi(z, s, a) & =\sum_{n=0}^{\infty} \frac{z^{n}}{(n+a)^{s}} \\
& =\frac{1}{\Gamma(s)} \int_{0}^{\infty} \frac{t^{s-1} e^{-a t}}{1-z e^{-t}} d t
\end{aligned}
$$

with $\Re(a)>0$; $\Re(s)>0$ when $|z| \leq 1(z \neq 1) ; \Re(s)>1$ when $|z|=1$ is the Hurwitz-Lerch zeta function, $c f$. [8] and [21]. Some special cases of $\Phi(z, s, a)$ are listed as follows:

- the Riemann zeta function

$$
\Phi(1, s, 1)=\zeta(s) \quad(\Re(s)>1)
$$


- the Euler-zeta function

$$
\Phi(-1, s, 1)=\zeta_{E}(s) \quad(\Re(s)>0)
$$

- the polylogarithm function

$$
z \Phi(z, k, 1)=L i_{k}(z)
$$

see [1] and [22] for details.

Hence, we state the following corollary.

\section{Corollary 2.4 The following equality holds true:}

$$
\eta_{1, u}(s)=(1-u)^{s} \Phi(u, s+1,1) .
$$

In view of the calculations above, we observe that $\eta_{k, u}(s)$ is a holomorphic function for $\Re(s)>0$ because of the comparison test as $e_{k}(\log (1+(1-u) t)) \leq e_{1}(\log (1+(1-u) t))$ with the assumption $(1-u) t \geq 0$. By (17), we have

$$
\begin{aligned}
\eta_{k, u}(s)= & \frac{(1-u)^{s-1}}{\Gamma(s)} \int_{0}^{\infty} \frac{t^{s-1}}{e^{t}-u} e_{k}(\log (1+(1-u) t)) d t \\
= & \frac{(1-u)^{s-1}}{\Gamma(s)} \int_{0}^{1} \frac{t^{s-1}}{e^{t}-u} e_{k}(\log (1+(1-u) t)) d t \\
& +\frac{(1-u)^{s-1}}{\Gamma(s)} \int_{1}^{\infty} \frac{t^{s-1}}{e^{t}-u} e_{k}(\log (1+(1-u) t)) d t .
\end{aligned}
$$

The second integral in (19) converges absolutely for any $s \in \mathbb{C}$, and thus the second term on the right-hand side vanishes at nonpositive integers. Hence, we get

$$
\lim _{s \rightarrow-m}\left|\frac{(1-u)^{s-1}}{\Gamma(s)} \int_{1}^{\infty} \frac{t}{e^{t}-u} e_{k}(\log (1+(1-u) t)) d t\right| \leq \frac{(1-u)^{-m-1}}{\Gamma(-m)} M=0
$$

since

$$
\Gamma(s) \Gamma(1-s)=\frac{\pi}{\sin (\pi s)} .
$$

Moreover, for $\Re(s)>0$, the first integral in (19) can be written as

$$
\begin{aligned}
& \frac{(1-u)^{s-1}}{\Gamma(s)} \int_{0}^{1} \frac{e_{k}(\log (1+(1-u) t))}{e^{t}-u} t^{s-1} d t \\
& =\frac{(1-u)^{s-1}}{\Gamma(s)} \sum_{n=0}^{\infty} \frac{G_{n}^{(F, k)}(u)}{n !} \int_{0}^{1} t^{n+s-1} d t \\
& =\frac{(1-u)^{s-1}}{\Gamma(s)} \sum_{n=0}^{\infty} \frac{G_{n}^{(F, k)}(u)}{n !} \frac{1}{n+s},
\end{aligned}
$$


which defines an entire function of $s$. Hence, we obtain that $\eta_{k, u}(s)$ can be continued to an entire function of $s$. From (19) and (20), we attain

$$
\begin{aligned}
\eta_{k, u}(-m) & =\lim _{s \rightarrow-m} \frac{(1-u)^{s-1}}{\Gamma(s)} \int_{0}^{1} \frac{e_{k}(\log (1+(1-u) t))}{e^{t}-u} t^{s-1} d t \\
& =\lim _{s \rightarrow-m} \frac{(1-u)^{s-1}}{\Gamma(s)} \sum_{n=0}^{\infty} \frac{G_{n}^{(F, k)}(u)}{n !(n+s)} \\
& =\cdots+0+\cdots+0+\lim _{s \rightarrow-m} \frac{(1-u)^{s-1}}{\Gamma(s)} \frac{G_{m}^{(F, k)}(u)}{m !(m+s)}+0+0+\cdots \\
& =\lim _{s \rightarrow-m} \frac{(1-u)^{s-1}}{m+s} \frac{\Gamma(1-s) \sin (\pi s)}{\pi} \frac{G_{m}^{(F, k)}(u)}{m !} \\
& =(1-u)^{-m-1} \Gamma(1+m) \cos (\pi m) \frac{G_{m}^{(F, k)}(u)}{m !} \\
& =(1-u)^{-m-1}(-1)^{m} G_{m}^{(F, k)}(u) .
\end{aligned}
$$

Thus, we get the following theorem.

Theorem 2.3 Let $k \in \mathbb{N}$. The function $\eta_{k, u}(s)$ has an analytic continuation to a function of $s \in \mathbb{C}$, and the special values at nonpositive integers are given by

$$
\eta_{k, u}(-m)=(1-u)^{-m-1}(-1)^{m} G_{m}^{(F, k)}(u) \quad\left(m \in \mathbb{N}_{0}\right) .
$$

Taking $k=1$ in Theorem 2.3 and by (18), we have the following corollary.

Corollary 2.5 The following identity holds true:

$$
\Phi(u,-m+1,1)=\frac{(-1)^{m}}{1-u} G_{m}^{F}(u) .
$$

Corollary 2.6 Upon setting $k=1$ and $u=-1$ in Theorem 2.3 we arrive at

$$
\zeta_{E}(1-m)=\frac{(-1)^{m}}{2} G_{m}(u)
$$

The following derivative property holds true ( $c f .[12])$ :

$$
\frac{d}{d x} e_{k}(x)=\frac{1}{x} e_{k-1}(x)
$$

and the following integral representation also holds true for $k>1$ :

$$
e_{k}(x)=\int_{0}^{x} \frac{1}{t} \underbrace{\int_{0}^{t} \frac{1}{t} \cdots \int_{0}^{t} \frac{1}{t}}_{(k-2) \text { times }}\left(e^{t}-1\right) d t d t \cdots d t .
$$

Now, we give the following theorem. 
Theorem 2.4 For $n \in \mathbb{N}_{0}$, we have

$$
G_{n}^{(F, 2)}(u)=\sum_{l=0}^{n}\left(\begin{array}{l}
n \\
l
\end{array}\right)(1-u)^{l} \frac{B_{l}^{(l)}}{l+1} G_{n-l}^{F}(u) .
$$

Proof By (22), we first consider that

$$
\begin{aligned}
\frac{d}{d x} e_{k}(\log (1+(1-u) x)) & =\sum_{n=1}^{\infty} \frac{(\log (1+(1-u) x))^{n}}{(n-1) ! n^{k}} \\
& =\frac{1-u}{1+(1-u) x} \sum_{n=1}^{\infty} \frac{(\log (1+(1-u) x))^{n-1}}{(n-1) ! n^{k-1}} \\
& =\frac{1-u}{(1+(1-u) x) \log (1+(1-u) x)} e_{k-1}(\log (1+(1-u) x))
\end{aligned}
$$

From (23) and (24), for $k>1$, we can write

$$
\begin{aligned}
\sum_{n=0}^{\infty} G_{n}^{(F, k)}(u) \frac{t^{n}}{n !} \\
=\frac{(1-u)^{k-1}}{e^{t}-u} \int_{0}^{x} \frac{1}{(1+(1-u) t) \log (1+(1-u) t)} \\
\quad \times \underbrace{\int_{0}^{t} \frac{1}{(1+(1-u) t) \log (1+(1-u) t)} \cdot \int_{0}^{t} \frac{(1-u) t}{(1+(1-u) t) \log (1+(1-u) t)}}_{(k-2) \text { times }} d t d t \cdots d t .
\end{aligned}
$$

Hence, we acquire

$$
\begin{aligned}
\sum_{n=0}^{\infty} G_{n}^{(F, 2)}(u) \frac{x^{n}}{n !} & =\frac{1-u}{e^{x}-u} \int_{0}^{x} \frac{(1-u) t}{(1+(1-u) t) \log (1+(1-u) t)} d t \\
& =\frac{1-u}{e^{x}-u} \int_{0}^{x} \sum_{n=0}^{\infty}(1-u)^{n} B_{n}^{(n)} \frac{t^{n}}{n !} d t \\
& =\frac{(1-u) x}{e^{x}-u} \sum_{n=0}^{\infty} \frac{(1-u)^{n} B_{n}^{(n)}}{n+1} \frac{x^{n}}{n !} \\
& =\left(\sum_{n=0}^{\infty} G_{n}^{F}(u) \frac{x^{n}}{n !}\right)\left(\sum_{n=0}^{\infty} \frac{(1-u)^{n} B_{n}^{(n)}}{n+1} \frac{x^{n}}{n !}\right) \\
& =\sum_{n=0}^{\infty}\left(\sum_{l=0}^{n}\left(\begin{array}{c}
n \\
l
\end{array}\right)(1-u)^{l} \frac{B_{l}^{(l)}}{l+1} G_{n-l}^{F}(u)\right) \frac{x^{n}}{n !} .
\end{aligned}
$$

Thus, we have

$$
G_{n}^{(F, 2)}(u)=\sum_{l=0}^{n}\left(\begin{array}{l}
n \\
l
\end{array}\right)(1-u)^{l} \frac{B_{l}^{(l)}}{l+1} G_{n-l}^{F}(u) .
$$

This finalizes the proof of the theorem. 


\section{The unipoly-Frobenius-Genocchi polynomials}

Motivated and inspired by the definition of the unipoly-Bernoulli polynomials in (10) given by Kim and Kim [12], we introduce unipoly-Frobenius-Genocchi polynomials by means of the unipoly function attached to $p$ given in (9) as follows:

$$
\sum_{n=0}^{\infty} G_{n, p}^{(F, k)}(x ; u) \frac{t^{n}}{n !}=\frac{u_{k}(\log (1+(1-u) t) \mid p)}{e^{t}-u} e^{x t}
$$

Note that taking $x=0$ in $(25), G_{n, p}^{(F, k)}(0 ; u):=G_{n, p}^{(F, k)}(u)$ are called the unipoly-FrobeniusGenocchi numbers.

By (25), we consider that

$$
\begin{aligned}
\sum_{n=0}^{\infty} G_{n, p}^{(F, k)}(x ; u) \frac{t^{n}}{n !} & =\frac{u_{k}(\log (1+(1-u) t) \mid p)}{e^{t}-u} e^{x t} \\
& =\sum_{n=0}^{\infty} G_{n, p}^{(F, k)}(u) \frac{t^{n}}{n !} \sum_{n=0}^{\infty} \frac{x^{n} t^{n}}{n !} \\
& =\sum_{n=0}^{\infty}\left(\sum_{l=0}^{n}\left(\begin{array}{c}
n \\
l
\end{array}\right) G_{n-l, p}^{(F, k)}(u) x^{l}\right) \frac{t^{n}}{n !} .
\end{aligned}
$$

Hence, we give the following theorem.

\section{Theorem 3.1 The following relation}

$$
G_{n, p}^{(F, k)}(x ; u)=\sum_{l=0}^{n}\left(\begin{array}{l}
n \\
l
\end{array}\right) G_{n-l, p}^{(F, k)}(u) x^{l}
$$

is true for $k \in \mathbb{Z}$ and $n \in \mathbb{N}_{0}$.

We observe that

$$
\begin{aligned}
\sum_{n=0}^{\infty} \frac{d}{d x} G_{n, p}^{(F, k)}(x ; u) \frac{t^{n}}{n !} & =\frac{u_{k}(\log (1+(1-u) t) \mid p)}{e^{t}-u} \frac{d}{d x} e^{x t} \\
& =\sum_{n=0}^{\infty} G_{n, p}^{(F, k)}(x ; u) \frac{t^{n+1}}{n !} .
\end{aligned}
$$

Therefore, we give the following theorem.

Theorem 3.2 Let $k \in \mathbb{Z}$ and $n \in \mathbb{N}_{0}$. We have the following derivative rule:

$$
\frac{d}{d x} G_{n, p}^{(F, k)}(x ; u)=n G_{n-1, p}^{(F, k)}(x ; u) .
$$

By Theorem 3.2, we consider that

$$
\int_{\alpha}^{\beta} G_{n, p}^{(F, k)}(x ; u) d x=\frac{1}{n+1} \int_{\alpha}^{\beta} \frac{d}{d x} G_{n+1, p}^{(F, k)}(x ; u) d x=\frac{G_{n+1, p}^{(F, k)}(\beta ; u)-G_{n+1, p}^{(F, k)}(\alpha ; u)}{n+1} .
$$

Thus, we provide the following theorem. 
Theorem 3.3 Let $k \in \mathbb{Z}$ and $n \in \mathbb{N}_{0}$. We have the following integral rule:

$$
\int_{\alpha}^{\beta} G_{n, p}^{(F, k)}(x ; u) d x=\frac{G_{n+1, p}^{(F, k)}(\beta ; u)-G_{n+1, p}^{(F, k)}(\alpha ; u)}{n+1} .
$$

Upon setting $p(n)=\frac{1}{\Gamma(n)}$ in (25), we acquire

$$
\begin{aligned}
\sum_{n=0}^{\infty} G_{n, \frac{1}{\Gamma}}^{(F, k)}(u) \frac{t^{n}}{n !}= & \frac{1}{e^{t}-u} u_{k}\left(\log (1+(1-u) t) \mid \frac{1}{\Gamma}\right) \\
= & \frac{1}{e^{t}-u} \sum_{m=1}^{\infty} \frac{(\log (1+(1-u) t))^{m}}{m^{k}(m-1) !} \\
= & \frac{1}{e^{t}-u} e_{k}(\log (1+(1-u) t)) \\
& \times \sum_{n=0}^{\infty} G_{n}^{(F, k)}(u) \frac{t^{n}}{n !},
\end{aligned}
$$

which gives the following relation:

$$
G_{n, \frac{1}{\Gamma}}^{(F, k)}(u)=G_{n}^{(F, k)}(u) .
$$

From (9) and (25), we have

$$
\begin{aligned}
\sum_{n=0}^{\infty} & G_{n, p}^{(F, k)}(u) \frac{t^{n}}{n !} \\
& =\frac{1}{e^{t}-u} \sum_{m=1}^{\infty} \frac{p(m)}{m^{k}}(\log (1+(1-u) t))^{m} \\
& =\frac{1}{e^{t}-u} \sum_{m=0}^{\infty} \frac{p(m+1)(m+1) !}{(m+1)^{k}} \frac{(\log (1+(1-u) t))^{m+1}}{(m+1) !} \\
& =\frac{1}{e^{t}-u} \sum_{m=0}^{\infty} \frac{p(m+1)(m+1) !}{(m+1)^{k}} \sum_{n=m+1}^{\infty} S_{1}(n, m+1)(1-u)^{n} \frac{t^{n}}{n !} \\
& =\frac{(1-u) t}{e^{t}-u} \sum_{m=0}^{\infty} \frac{p(m+1)(m+1) !}{(m+1)^{k}} \sum_{n=m}^{\infty} S_{1}(n+1, m+1)(1-u)^{n} \frac{t^{n}}{(n+1) !} \\
& =\sum_{n=0}^{\infty} G_{n}^{F}(u) \frac{t^{n}}{n !} \sum_{n=0}^{\infty}\left(\sum_{m=0}^{n} \frac{p(m+1)(m+1) !}{(m+1)^{k}} \frac{S_{1}(n+1, m+1)}{n+1}(1-u)^{n}\right) \frac{t^{n}}{n !} \\
& =\sum_{n=0}^{\infty}\left(\sum_{l=0}^{n} \sum_{m=0}^{l}\left(\begin{array}{l}
n \\
l
\end{array}\right) \frac{p(m+1)(m+1) !}{(m+1)^{k}} \frac{S_{1}(l+1, m+1)}{l+1}(1-u)^{l} G_{n-l}^{F}(u)\right) \frac{t^{n}}{n !},
\end{aligned}
$$

which yields the following theorem.

Theorem 3.4 For $k \in \mathbb{Z}$ and $n \in \mathbb{N}_{0}$, we have

$$
G_{n, p}^{(F, k)}(u)=\sum_{l=0}^{n} \sum_{m=0}^{l}\left(\begin{array}{l}
n \\
l
\end{array}\right) \frac{p(m+1)(m+1) !}{(m+1)^{k}} \frac{S_{1}(l+1, m+1)}{l+1}(1-u)^{l} G_{n-l}^{F}(u) .
$$


Particularly, for $p(n)=\frac{1}{\Gamma(n)}$,

$$
G_{n, \frac{1}{T}}^{(F, k)}(u)=\sum_{l=0}^{n} \sum_{m=0}^{l}\left(\begin{array}{l}
n \\
l
\end{array}\right) \frac{m+1}{(m+1)^{k}} \frac{S_{1}(l+1, m+1)}{l+1}(1-u)^{l} G_{n-l}^{F}(u) .
$$

\section{Conclusion}

Motivated by the definition of the type 2 poly-Bernoulli polynomials introduced by Kim and Kim [12], we have considered a class of new generating functions for the Frobenius-Genocchi polynomials, called the type 2 poly-Frobenius-Genocchi polynomials, by means of the polyexponential function as follows:

$$
\sum_{n=0}^{\infty} G_{n}^{(F, k)}(x ; u) \frac{t^{n}}{n !}=\frac{e_{k}(\log (1+(1-u) t))}{e^{t}-u} e^{x t}
$$

Then, we have derived some useful relations and properties. We have showed that the type 2 poly-Frobenius-Genocchi polynomials equal a linear combination of the classical Frobenius-Genocchi polynomials and Stirling numbers of the first kind. Equation (29) enables us to find some new identities of the usual Genocchi polynomials in the case when $k=-u=1$.

Moreover, inspired by the definition of the unipoly-Bernoulli polynomials introduced by Kim and Kim [12] we have introduced the unipoly-Frobenius-Genocchi polynomials by means of a unipoly function as follows:

$$
\sum_{n=0}^{\infty} G_{n, p}^{(F, k)}(x ; u) \frac{t^{n}}{n !}=\frac{u_{k}(\log (1+(1-u) t) \mid p)}{e^{t}-u} e^{x t}
$$

By using this generating function, we have given multifarious properties including unipoly-Frobenius-Genocchi polynomials and the classical Frobenius-Genocchi polynomials.

\section{Acknowledgements}

The authors would like to thank the reviewers for their valuable suggestions and comments, which have improved the presentation of the paper substantially.

\section{Funding}

This research received no external funding.

Availability of data and materials

Not applicable.

Competing interests

The authors declare that they have no competing interests.

Authors' contributions

All authors contributed equally to this article. All authors read and approved the final manuscript.

\section{Author details}

'Department of Basic Sciences of Engineering, Faculty of Engineering and Natural Sciences, Iskenderun Tecnical University, TR-31200 Hatay, Turkey. ${ }^{2}$ Department of Mathematics, Faculty of Arts and Science, University of Gaziantep, TR-27310 Gaziantep, Turkey. ${ }^{3}$ Department of Economics, Faculty of Economics, Administrative and Social Sciences, Hasan Kalyoncu University, TR-27410 Gaziantep, Turkey.

\section{Publisher's Note}

Springer Nature remains neutral with regard to jurisdictional claims in published maps and institutional affiliations. 
References

1. Acikgoz, M., Simsek, Y.: On multiple interpolation functions of the Nörlund-type $q$-Euler polynomials. Abstr. Appl. Anal. 2009, Article ID 382574 (2009)

2. Araci, S.: Novel identities for q-Genocchi numbers and polynomials. J. Funct. Spaces Appl. 2012, Article ID 214961 (2012)

3. Araci, S.: Novel identities involving Genocchi numbers and polynomials arising from applications of umbral calculus. Appl. Math. Comput. 233, 599-607 (2014)

4. Araci, S., Acikgoz, M., Sen, E.: On the von Staudt-Clausen's theorem associated with $q$-Genocchi numbers. Appl. Math. Comput. 247, 780-785 (2014)

5. Cangul, I.N., Kurt, V., Ozden, H., Simsek, Y.: On the higher-order w-q-Genocchi numbers. Adv. Stud. Contemp. Math. (Kyungshang) 19, 39-57 (2009)

6. Dolgy, D.V., Jang, L.C.: Some identities on the poly-Genocchi polynomials and numbers. Symmetry 12, 1007 (2020)

7. Duran, U., Acikgoz, M., Araci, S.: Symmetric identities involving weighted q-Genocchi polynomials under S4. Proc. Jangjeon Math. Soc. 18, 445-465 (2015)

8. Gaboury, S., Bayad, A.: Series representations at special values of generalized Hurwitz-Lerch zeta function. Abstr. Appl. Anal. 2013, Article ID 975615 (2013)

9. He, Y., Kim, T.: General convolution identities for Apostol-Bernoulli, Euler and Genocchi polynomials. J. Nonlinear Sci. Appl. 9, 4780-4797 (2016)

10. Khan, N., Usman, T., Nisar, K.S.: A study of generalized Laguerre poly-Genocchi polynomials. Mathematics 7, 219 (2019)

11. Khan, W.A., Srivastava, D.: On the generalized Apostol-type Frobenius-Genocchi polynomials. Filomat 33(7), 1967-1977 (2019)

12. Kim, D.-S., Kim, T.: A note on polyexponential and unipoly functions. Russ. J. Math. Phys. 26(1), 40-49 (2019)

13. Kim, T.: Some identities for the Bernoulli, the Euler and the Genocchi numbers and polynomials. Adv. Stud. Contemp. Math. 20, 23-28 (2010)

14. Kim, T., Kim, D.S.: Degenerate polyexponential functions and degenerate Bell polynomials. J. Math. Anal. Appl. 487(2), $124017(2020)$

15. Kim, T., Kim, D.S., Kwon, J.K., Kim, H.Y.: A note on degenerate Genocchi and poly-Genocchi numbers and polynomials. J. Inequal. Appl. 2020, $110(2020)$

16. Kim, T., Kim, D.S., Kwon, J.K., Lee, H.S.: Degenerate polyexponential functions and type 2 degenerate poly-Bernoulli numbers and polynomials. Adv. Differ. Equ. 2020, 168 (2020)

17. Kim, T., Rim, S.H., Dolgy, D.V., Lee, S.H.: Some identities of Genocchi polynomials arising from Genocchi basis. J. Inequal. Appl. 2013, Article ID 43 (2013)

18. Kumam, W., Srivastava, H.M., Wani, S.A., Araci, S., Kumam, P.: Truncated-exponential-based Frobenius-Euler polynomials. Adv. Differ. Equ. 2019, 530 (2019)

19. Kurt, B., Simsek, Y.: On the Hermite based Genocchi polynomials. Adv. Stud. Contemp. Math. (Kyungshang) 23, 13-17 (2013)

20. Kwon, J., Jang, L.C.: A note on the type 2 poly-Apostol-Bernoulli polynomials. Adv. Stud. Contemp. Math. (Kyungshang) 30, 253-262 (2020)

21. Lin, S.-D., Srivastava, H.M.: Some families of the Hurwitz-Lerch zeta functions and associated fractional derivative and other integral representations. Appl. Math. Comput. 154(3), 725-733 (2004)

22. Srivastava, H.M., Choi, J.: Series Associated with the Zeta and Related Functions. Kluwer Academic, Dordrecht (2001)

23. Srivastava, H.M., Kurt, B., Simsek, Y.: Some families of Genocchi type polynomials and their interpolation functions. Integral Transforms Spec. Funct. 23, 919-938 (2012)

24. Wani, S.A., Khan, S., Nahid, T.: Gould-Hopper based Frobenius-Genocchi polynomials and their generalized form. Afr. Math. (2020). https://doi.org/10.1007/s13370-020-00804-2

25. Yasar, B.Y., Ozarslan, M.A.: Frobenius-Euler and Frobenius-Genocchi polynomials and their differential equations. New Trends Math. Sci. 3(2), 172-180 (2015)

\section{Submit your manuscript to a SpringerOpen ${ }^{\odot}$ journal and benefit from:}

- Convenient online submission

- Rigorous peer review

- Open access: articles freely available online

- High visibility within the field

- Retaining the copyright to your article

Submit your next manuscript at $>$ springeropen.com 